\title{
EFICACIA, TIEMPO Y CUMPLIMIENTO
}

«No conocemos exactamente qué motivos inducen a los hombres a cumplir las normas jurídicas. Ningún orden jurídico positivo ha sido nunca estudiado en una forma científica satisfactoria con el propósito de responder a esta pregunta».

Hans Kelsen

\section{I}

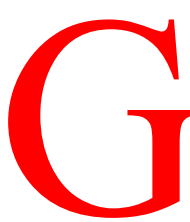
ran parte de las disputas del «positivismo jurídico» de este siglo han girado, explícita o implícitamente, acerca de la relación entre norma y conducta. Sin embargo, puede decirse que los resultados obtenidos son más bien pobres en relación a los esfuerzos empeñados en la disputa.

El análisis del cumplimiento de las normas es una manera de estudiar la esquiva relación arriba mencionada. En este trabajo expondré dos importantes sentidos del término «eficacia». También sugeriré la utilidad de trabajar el problema de la eficacia de las normas a partir de parámetros definidos. A la vez, mostraré algunas dificultades teóricas vinculadas a esta noción.

\section{II}

A menudo, las expresiones «cumplimiento» y «eficacia» son utilizadas por los juristas como sinónimas. Sin embargo, el término «eficacia» plantea algunos problemas de los que no somos conscientes cuando utilizamos el término «cumplimiento». Una razón de ello es que el universo del discurso que constituye el objeto de estudio de la ciencia jurídica está constituido por un conjunto de elementos a los que generalmente se asignan una cierta clase de propiedades. Una de tales propiedades es la denotada por el término «eficacia». ${ }^{1}$

Debido a la plurisignificatividad de «eficacia» surgen a menudo problemas de difícil solución ya que, por lo general, los juristas no precisan el sentido en que utilizan el término.

Para lo que aquí importa distinguiremos dos significados de eficacia ${ }^{2}$.

\footnotetext{
${ }^{1}$ Cf. Garzón Valdés, E.; 1977, pg. 65.

${ }^{2}$ Cf. Caracciolo, R.; 1977, pg. 179.
} 


\section{a) Eficacia como relación:}

Una norma (n) es eficaz cuando un estado de cosas (generalmente el resultado de una acción) se adecua al contenido normativo de (n). La norma (n) es eficaz respecto de los estados de cosas individuales miembros de la clase de estados de cosas genéricos prescriptos.

Para un conjunto finito de estados de cosas que deben realizarse de acuerdo a (n), la norma será eficaz cuando se establezca una relación de identidad entre la «conducta debida» y la «conducta producida» ${ }^{3}$, aun cuando sea ineficaz respecto de otra acción que no produce el resultado prescripto. Este significado de «eficacia» puede denominarse «eficacia (E1)». (En adelante (E1), por razones de brevedad).

En este primer sentido se precisa de una norma que prescriba una conducta humana, y de algún modo, es éste el uso primario del término «eficacia». ${ }^{4}$

Obviamente que la relación exigida por (E1) no puede ser satisfecha por normas que no prescriben comportamientos «... dado que las normas derogatorias no prescriben una cierta conducta y no pueden ser obedecidas y aplicadas como otras normas, ellas tampoco pueden ser inobservadas» ${ }^{5}$. La misma cuestión podría suscitarse con las normas permisivas, ya que desde una perspectiva semántica no es claro saber qué significa cumplir o incumplir un permiso para realizar una acción ${ }^{6}$.

\section{b) Eficacia como propiedad}

Este segundo sentido de «eficacia» denota una propiedad de una norma (n). Este significado puede denominarse «eficacia (E2)», y diremos que la norma (n) es eficaz (E2) cuando es posible afirmar verdaderamente que existen un número indeterminado de actos de cumplimiento de la norma (n). Aun cuando no sea del todo exacto, diremos que un acto (acción) cumple lo prescrito por una norma cuando el estado de cosas producido es idéntico al estado de cosas debido.

$\mathrm{Si}$ bien este segundo sentido de eficacia no es lógicamente independiente del primero, no por ello son equivalentes. Esta importante ambigüedad no siempre fue advertida; por ejemplo, Kelsen señala que identificar «validez» con «eficacia» es cometer la famosa «falacia naturalista» que dicho vulgarmente consiste en tomar como idénticas dos propiedades cualitativamente distintas ${ }^{7}$.

\footnotetext{
${ }^{3}$ Esto no significa que tal relación sea de carácter lógico. Lo que aquí importa es la posibilidad de predicar verdaderamente la equivalencia material entre un contenido normativo y la verdad de la descripción de un estado de cosas. Cf. Kelsen, H.; 1979, pg. 20.

${ }^{4}$ Cf. Kelsen, H.; 1949, pg. 41.

${ }^{5}$ Cf. Kelsen, H.; 1974, pg. 260.

${ }^{6}$ Vernengo, R.; 1983, pg. 440.

${ }^{7}$ Cf. Kelsen, H.; 1979, pg. 25 y Frankena, W.; 1974.
} 
Pero en algunos otros pasajes niega enfáticamente que la eficacia sea una cualidad de las normas ${ }^{8}$. La adscripción de la propiedad «eficacia» parece ser necesario en el caso de las normas consuetudinarias; Alchourron y Bulygin ${ }^{9}$ afirman que el enunciado «la norma (n) es eficaz» es analítico en el caso de que (n) sea una norma originada en la costumbre. Pero si no se discrimina entre la eficacia como relación de cumplimiento y la eficacia como propiedad, se tiene que la negación del enunciado en cuestión no es contradictorio en el supuesto de (E1). La razón es simple; en el sentido (E1) el enunciado «la norma (n) es eficaz» es sintético y por ende la negación no es contradictoria.

Polémica aparte, puede verse fácilmente que para que sea posible afirmar verdaderamente que la norma (n) es eficaz (E2) es necesario poder afirmar que la norma (n) es eficaz (E1). De ello se sigue que la verdad de la proposición expresada por el enunciado «la norma (n) es eficaz (E2)» es suficiente para decir que existen una cantidad indeterminada de estados de cosas que satisfacen lo prescrito por la norma (n).

¿Cuántos estados de cosas del tipo de eficacia (E1) son suficientes para la verdad de «la norma (n) es eficaz (E2)»? Una de las dificultades para responder a esta pregunta radica en la falta de precisión de las magnitudes o parámetros que se toman en cuenta en la elaboración de las respuestas.

Otra cuestión importante es la imposibilidad de independizar los juicios sobre la eficacia de las normas jurídicas de las teorías acerca de la naturaleza, estructura y función de las mismas normas. En otros términos, afirmar la verdad de los enunciados acerca de la eficacia de una norma o sistemas normativos presupone admitir una clase de enunciados que proporcionan los criterios de existencia e identidad de las normas jurídicas. Sin embargo, no se cuenta actualmente con un consenso general sobre el status científico y epistemológico de tales teorías ${ }^{10}$.

\section{III}

Gran número de resultado de acciones son estados de cosa y, normalmente, el resultado de una acción u omisión consiste en un cambio en el mundo. Si la relación entre acción y resultado es de índole conceptual ${ }^{11}$, entonces el tiempo empleado para ejecutar una acción es equivalente al tiempo empleado para alcanzar el resultado que define el tipo de acción en cuestión. Generalmente la complejidad (subjetiva y objetivamente

${ }^{8}$ Cf. Kelsen, H.; 1949, pg. 41 y Kelsen, H.; 1984, pg. 71.

${ }^{9}$ Alchourron, C. y Bulygin, E.; 1979, pg. 18.

${ }^{10}$ Véase como ejemplo relevante a los trabajos de Alchourron, C. y Bulygin, E. y los de Ota Weinberger, acerca de la naturaleza expresiva o hilética de las normas. (Ver los trabajos indicados en las referencias).

${ }^{11}$ Cf. von Wright, G.; 1970, pgs. 56 y 61. 
considerada) de la acción está en relación directa con el tiempo necesario para su ejecución; por ende, cumplir una norma implicará un consumo de tiempo proporcional a la complejidad de la acción exigida. Este segmento temporal puede denominarse «tiempo de cumplimiento» y su extensión depende de la naturaleza de la acción y la estructura de la norma. En muchos casos el contenido normativo no consiste en una acción sino en un conjunto de acciones a realizarse en ocasiones diferentes. El «tiempo de cumplimiento» será la suma del tiempo empleado en la ejecución de tales acciones. Antes de proseguir en este análisis es conveniente señalar algunas dificultades.

Se suele distinguir entre «reglas de hacer»y «reglas de ser» ${ }^{12}$. A estas últimas se las suele denominar «reglas ideales». No es difícil comprender que la manera de cumplir lo prescrito por estas reglas ideales (ser valiente, ser justo, etc.) es ejecutar una serie de actos de los que conforman los elementos de la clase de estados prescritos. Pareciera que en estos casos es bastante difícil, sin echar mano a criterios pragmáticos, justificar una respuesta acerca de la eficacia de una regla ideal; y por ende, establecer el tiempo necesario para cumplir lo prescrito por tales reglas. Es claro que ante las dudas planteadas por las reglas ideales se hace patente la ambigüedad del concepto de eficacia (E1); las reglas ideales no prescriben conductas sino estados de cosas. Este punto es especialmente importante para esclarecer las relaciones entre motivación normativa, eficacia y cumplimiento. Decir que se cumple una norma jurídica puede considerarse como la afirmación de la equivalencia material entre la verdad de la descripción de un contenido normativo y la verdad de la descripción de un estado de cosas. Supongamos que es obligatorio para un sujeto (s) realizar p\&q («\&» simboliza la conjunción). Ello equivale a la obligación de cambiar el mundo -p («-» simboliza la negación) a un mundo p, o mantener p si éste ya existe. Igualmente respecto de q. Si el mundo del sujeto (s) es -p\&q entonces el sujeto debe producir $\mathrm{p}$ y mantener q. Puede ser el caso de que el sujeto (s) produzca p pero q existe con independencia de lo que puede hacer (s), esto es: el sujeto (s) no puede hacer ni evitar $\mathrm{q}^{13}$. El sujeto (s) no produce la acción $\mathrm{p} \& \mathrm{q}$ aun cuando sea el caso de que es verdadero de que existe en el mundo el estado de cosas p\&q. El concepto de eficacia (E1) trata de reflejar lo que Kelsen denomina «eficacia normativa» ${ }^{14}$. Pero si la función principal del derecho es motivar comportamientos, sería conveniente tomar como concepto primario de eficacia a lo que Kelsen denomina «eficacia causal». La eficacia (E1) de una norma puede verse como una relación entre normas y acciones o entre normas y estados de cosas. En este trabajo, se mantendrá la definición propuesta: Una norma (n) es eficaz (E1)

\footnotetext{
${ }^{12}$ Cf. von Wright, G.; 1970, pg. 33 y von Wright, G.; 1976, pg. 43.

${ }^{13}$ Cf. von Wright, G.; 1970, pgs. 66 y 67.

${ }^{14}$ Kelsen, H.; 1970, pgs. 40 y 41.
} 
cuando un estado de cosas (generalmente el resultado de una acción) se adecua al contenido normativo de (n).

Otra dificultad, que simplemente mencionaremos, es la relación entre el tiempo de cumplimiento y la estructura y naturaleza de los mandatos de omitir, o las conjunciones o disyunciones de mandatos mixtos (hacer y omitir). ${ }^{15}$

La expresión «tiempo de cumplimiento» podría usarse (no sin provecho) ambiguamente en los siguientes sentidos.

a) El tiempo, definido en una norma, en que debe realizarse una acción.

b) El tiempo empleado en ejecutar una acción prescrita en una norma.

Para un conjunto de acciones que deben ejecutarse en un tiempo $t$, la norma que prescribe tal conducta define el segmento de tiempo relevante, asignando a las acciones debidas, que se ejecutan dentro del tiempo $t$ un valor positivo (cumplimiento), y clasifica a las demás situaciones resultantes que se producen fuera del tiempo $t$-aun cuando sean materialmente idénticas a las de t- con el valor complementario (incumplimiento) ${ }^{16}$.

La estructura de la norma determina, entre otras cosas, las condiciones relevantes para atribuir a una acción la calidad de conducta debida. Esto significa que es imprescindible saber cuál es la clase de sujetos que deben ejecutar la acción deónticamente calificada. No basta señalar que la función de las normas es motivar conductas sino que es necesario saber a quiénes se dirigen tales normas, porque las condiciones de ejecución de la acción, que se definirá como cumplimiento de la norma (n), variará significativamente si la norma (n) se dirige a los jueces, a los órganos aplicadores de la sanción, o a los ciudadanos en general ${ }^{17}$.

Al denotar el término «eficacia. (E1)» una relación entre la conducta debida y la conducta producida es obvio que la descripción del tiempo de cumplimiento permanece incierto hasta que no se explicita a quiénes se dirigen las prescripciones.

Lo expuesto hasta aquí sirve para mostrar que el tiempo de cumplimiento, que permite afirmar la existencia de eficacia (E1) (recordemos que un conjunto de relaciones de eficacia (E1) es condición necesaria para la verdad de los enunciados sobre la eficacia (E2) de una norma (n)), no puede establecerse por la simple lectura de las disposiciones legales sino que es necesario contar con un aparato conceptual que permita de terminar: (i) los sujetos normativos, (ii) las características de las acciones debidas y (iii) el tiempo en que deben ejecutarse. Obviamente, estos tres tópicos deben ser resueltos mediante el análisis del concepto de «norma».

${ }^{15}$ Cf. Nino, C.; 1987, pg. 95 y Guibourg, R.; 1985, pg. 17.

${ }^{16}$ Kelsen, H.; 1979, pg. 34, nota 15.

${ }^{17}$ Cf. Kelsen, H.; 1969, pg. 34. Kelsen, H.; 1949, pg. 63. Capella, J.; 1968, pgs. 134 y 135 y Ross, A., 1963, pg. 35. 
Desgraciadamente aun no contamos con teorías paradigmáticas acerca de esta heterogénea clase de objeto.

\section{IV}

Es frecuente atribuir a una norma la propiedad de ser eficaz (E2) cuando «la mayoría de los sujetos en la mayoría de las ocasiones obedecen las normas», o «cuando la desobediencia no es habitual» ${ }^{18}$; cuando existe un mínimum de adecuación entre lo que es y lo que debe ser. Pero todas estas caracterizaciones, de patente vaguedad, pueden tornarse circulares si no se identifican parámetros que definan un contexto en los que términos como «mayoría», y «habitual» sean operativos. Los parámetros en cuestión pueden ser de naturaleza espacial, personal o temporal. Respecto de este último, los juristas operan, por lo general, con la totalidad del tiempo de existencia de una norma.

Al enfrentar un interrogante sobre la eficacia (E2) de una norma (n), les interesa establecer un número indeterminado de actos de cumplimiento desde que la norma entró «en vigor» hasta el momento en que surge el interrogante. Pero de ningún modo es claro cuáles son las condiciones que deben satisfacerse para afirmar verdaderamente la existen cia de una norma. Una norma (n) puede «existir» (pertenecer) en un sistema S y no en un sistema Sn. A su vez la norma (n) puede «existir» (ser aplicable) en Sn y no en S. El tiempo de existencia de una norma puede analizarse en función de la membrecía (tiempo externo) o de la aplicabilidad (tiempo interno) $)^{19}$.

Probablemente estas complicaciones teóricas sean la razón de que los juristas se limiten a exponer sólo tres o cuatro ejemplos triviales y trillados de normas ineficaces que, por definición, son absolutamente irrelevantes para la vida cotidiana.

Un análisis cuidadoso del problema de la eficacia (E2) permite ver la utilidad de diferenciar grupos de personas o conjuntos de ocasiones que puedan servir de base a la pregunta ¿Por qué la norma (n) tuvo un alto grado de acatamiento en el tiempo t y no ocurrió de igual modo en el tiempo tn?

Supongamos un sujeto (s) y una sucesión de ocasiones para ejecutar la acción prescrita por la norma (n).

Diag. I: (n); (s)

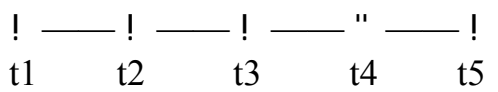

\footnotetext{
${ }^{18}$ von Wright, G.; 1970, pgs. 139 y 140.

${ }^{19}$ Bulygin, E; 1982, pg. 67.
} 
Los círculos llenos simbolizan actos de cumplimiento del sujeto (s), por ello es posible decir que (n) es eficaz (E1) en $\mathrm{t} 1, \mathrm{t} 2$, $\mathrm{t} 3 \mathrm{y} \mathrm{t} 5$, y que es ineficaz (E1) en t4. En esta historia no hay problemas en decir que la norma (n) es eficaz (E2), aun cuando sea verdadero que (n) no es eficaz (E1) en $t 4$.

Diag. II: (n); (s)

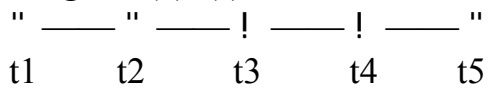

En este caso la situación es más compleja. El sujeto (s) mediante su acción en t3 y t4 conforman los hechos que hacen verdadero el enunciado «(n) es eficaz (E1) en t3 y t4» pero ¿es (n) eficaz (E2)? Si tomamos como criterio de asignación de eficacia (E2) al hecho de que la norma es obedecida en «la mayoría de las ocasiones», y tomamos en cuenta la sucesión de ocasiones de t1 a t5 podemos decir que (n) no es eficaz (E2) porque en la mayoría de las ocasiones fue desobedecida. Pero podría suceder que el segmento temporal escogido sea el que va desde $\mathrm{t} 3$ a $\mathrm{t} 5 \mathrm{y}$, en ese caso, sería verdadero decir que (n) es eficaz (E2).

Tal vez ocurre que el Diag. II representa un segmento temporal determinado por la pertenencia de (n) a un sistema Sn que se configura en un tiempo posterior al indicado por la pertenencia de (n) a un sistema $\mathrm{S}$.

Diag. III: (n); (s)

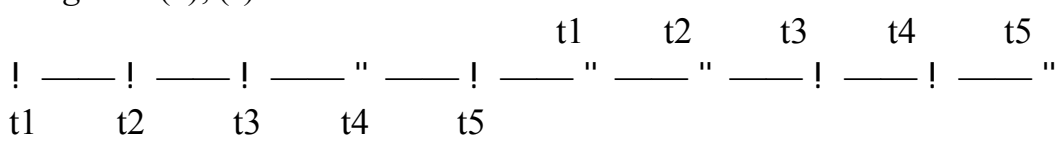

Al sumarse los segmentos temporales, la afirmación «(n) es eficaz (E2)» que será falsa sobre la base del diag. II, se torna verdadera ya que en la mayoría de las ocasiones se realiza la conducta prescrita. Observando los diagramas expuestos notamos claramente las diferentes respuestas posibles a la pregunta por la eficacia (E2) de la norma (n); y la verdad de tales respuestas es relativa a los sistemas normativos, o bien a los segmentos temporales que se tomen como referencia.

Supongamos que en la búsqueda de una explicación acerca de la oscilación de la eficacia (E1) de una norma en su historia, logramos identificar un estado de cosas p tal que siempre que p ocurre entonces se realiza la conducta debida. En este supuesto podríamos decir que el estado de cosas $\mathrm{p}$ es condición suficiente de la realización de la conducta prescrita. Quizás nunca sea posible establecer con exactitud las condiciones suficientes de ciertos comportamientos humanos, pero no cabe duda que 
el «análisis causal» constituye una poderosa herramienta para el aprendizaje de la motivación de conductas ${ }^{20}$.

Un jurista preocupado por la relación entre norma y conducta procurará establecer cuáles son las razones que sirven para explicar la oscilación de una norma en su historia ${ }^{21}$.

Si tal tarea es llevada a cabo con éxito, entonces será posible utilizar racionalmente al sistema normativo para explicar conductas (esquema de interpretación) y para producir determinados resultados (esquema de transformación).

Tradicionalmente se ha hecho hincapié en que la relación entre validez y eficacia es uno de los problemas fundamentales de una teoría positivista del derecho ${ }^{22}$. Esto contrasta abiertamente con el hecho de que los usuarios directos de los sistemas normativos (jueces, abogados, etc.) rara vez tienen en cuenta los problemas que tal relación implica. Tal vez por ello sea conveniente poner más énfasis en la eficacia (E1) como un dato del funcionamiento del sistema más que un requisito de pertenencia. En esta perspectiva, la división del tiempo en segmentos permite relativizar la importancia de la eficacia como propiedad y señala marcos relevantes para el análisis de las causas de la acción humana, poniendo el acento en la eficacia como relación; o mejor dicho, en la problemática del cumplimiento de las normas.

Por último hay que poner de manifiesto que en lo expuesto, el tiempo «real» existente entre los círculos de los diagramas pueden ser de días, horas o años, y depende más de la estructura y función de las normas jurídicas que de un dato de la realidad.

\section{AGRADECIMIENTOS}

Agradezco a Ricardo Caracciolo, Ricardo Guibourg, Gustavo Cosacov y Cristina Redondo, por la lectura de los originales de este trabajo y por sus valiosas sugerencias.

\footnotetext{
${ }^{20}$ Cf. von Wright, G.; 1979, pg. 78.

${ }^{21}$ Cf. Ross, A.; 1963, pg. 53.

${ }^{22}$ Cf. Kelsen, H.; 1979, pg. 219.
} 


\section{REFERENCIAS}

ALCHOURRON, C. y BULYGIN, E. «Sobre la existencia de las normas jurídicas», 1979. Valencia (Venezuela).

«The expressive conception of norms» en New studies in deontic logic, Hilpinen, R. (ed.). Pgs. 95-124. Reidel Publishing Company, 1981.

«The pragmatic foundation for a logic of norms» en Rechtstheorie, 15 (1984). Pg. 453-464. BULYGIN, E.: «Tirne and validity» en Deontic logic, computational linguistics and legal information systems, Martino, A (ed.). Vol. II. Pgs. 65-8 1. North Holland Publishing Company. 1982. «Norms and logic» en Law and philosophy 4 (1985). Pgs. 145-163. Reidel Publishing Company.

CAPELLA, J.: «El derecho como lenguaje». 1968. Ariel. Barcelona.

CARACCIOLO, R.: «Interpretación de sentido y conocimiento jurídico» en Revista latinoamericana de filosofía. Pgs. 177-184. Vol. III. n. ${ }^{\circ} 2.1977$.

FRANKENA, W.: «La falacia naturalista» en Teorías sobre la ética, compilación de P. Foot. Fondo de cultura económica. México, 1974. Trad. de M. Arboli.

(«The Naturalistic Fallacy» en Theories of ethics. 1967. Oxford University Press, Londres).

GARZÓN VALDÉS, E.: «Algunos modelos de validez normativa» en Revista latinoamericana de filosofía. Pgs. 41-68. Vol. III. N. ${ }^{\circ} 1.1977$.

GUIBOURG, R.: «Some reflections about the concept of action» en Archiv für Recths und Sozialphilosophie. LXXI/Heft I 1. Quartal. 1985.

KELSEN, H.: «Teoría general del derecho y del estado». 1949. UNAM. México. Trad. de E. García Maynez.

(«General Theory of law and state». 1945. Harvard University Press. USA.). Argentina.

«Contribuciones a la teoría pura del derecho». 1969. Centro editor de América Latina.

«Derogación» en Boletín mexicano de derecho comparado. N. ${ }^{\circ}$ 21. 1974. Trad. de A. Ortiz. «Teoría Pura del derecho». 1979. UNAM. México. Trad. de R. Vernengo.

(«Reine Rechtsehere». 1960. F. Deuticke. Viena).

Bonavidez.

«Kelsen-Klug: Normas jurídicas e analise logica». 1984. Forense. Brasil. Trad. de P.

(«Rechtsnormen und logische analyse». 1981. F. Deuticke, Viena).

NINO, C.: «Introducción a la filosofía de la acción humana». 1987. EUDEBA. Argentina.

ROSS, A.: «Sobre el derecho y la justicia». 1963. EUDEBA. Argentina. Trad. de G. Carrió. («On law and justice». 1958. University of California Press. Berkeley. USA.).

VERNENGO, R.: «Sistemas normativos dinámicos y la idea de libertad jurídica» en $E l$ lenguaje del derecho. Homenaje a Genaro Carrió. Pgs. 453-443. Abeledo Perrot. Argentina. 1983. 
WEINBERGER, O.: «On the meaning of norm sentences, normative inconsistency, and normative entailment. A reply to Carlos Alchourron and Eugenio Bulygin» en Rechtstheorie, 15 (1984). de G. Ferrero.

von WRIGHT, G.: «Norma y Acción: Una investigación lógica» 1970. Tecnos. Madrid. Trad.

(«Norm and action: A logical enquiry». 1963. Routledge \& Keagan Paul. Londres).

«Un ensayo de lógica deóntica y la teoría general de la acción». 1976. UNAM. México. Trad. de E. Garzón Valdez.

(«An Essay in deontic logic and the general theory of action». 1968. Acta Filosófica Fenica. North Holland).

«Explicación y Comprensión». 1979. Alianza Universidad. Madrid. Trad. de V. Renón. («Explanation and understanding». 1971. Routledge \& Keagan Paul. Londres.). 